\title{
Lymphatische Neoplasien bei jungen Patienten
}

\author{
Peter Borchmann $^{\mathrm{a}} \quad$ Nicola Gökbuget ${ }^{\mathrm{b}} \quad$ Gerald Wulf $^{c} \quad$ Lorenz Trümper $^{\mathrm{c}}$

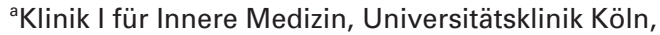 \\ ${ }^{b}$ Medizinische Klinik II, Klinikum der Goethe-Universität Frankfurt/Main,

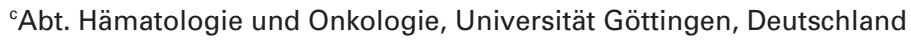

\section{Die AYA-Problematik am Beispiel der akuten Iymphatischen Leukämie \\ Verantwortliche Autorin: Nicola Gökbuget, Frankfurt/Main}

Die akute lymphatische Leukämie (ALL) wird häufig als «klassische» Erkrankung Heranwachsender und junger Erwachsener (Adolescents and Young Adults (AYA)) bezeichnet, was durch die tatsächlichen Fallzahlen jedoch nicht bestätig wird. Angaben zur Häufigkeit verschiedener Tumorentitäten sind etwa dem Surveillance, Epidemiology and End Results (SEER)-Programm der USA zu entnehmen [1, 2]. In den Jahren 1975-2000 waren von den jungen Krebspatienten im Alter zwischen 15 und 29 Jahren lediglich 6\% an Leukämien erkrankt. Auf die ALL entfielen in dieser Altersgruppe nur 2,1\% der Krebserkrankungen (Tab. 1).

Auch die Inzidenz der ALL ist im Adoleszenten-Alter nicht besonders hoch. Der Inzidenzgipfel liegt vielmehr bei Kindern im Alter von unter 5 Jahren. Danach fällt die Inzidenz stetig und steigt erst ab einem Lebensalter von etwa 55 Jahren wieder an.

ALL-Patienten im Alter zwischen 15 und 18 Jahren, zum Teil bis 21 Jahren, wurden in der Vergangenheit in verschiedenen Ländern sowohl in Zentren für pädiatrische Hämatologie und Onkologie als auch in der internistischen Hämatologie behandelt und jeweils in entsprechende Studien eingebracht. Die Zuordnung der Patienten in die Pädiatrie oder die Erwachsenen-Hämatologie erfolgt entweder zufällig oder in Abhängigkeit von den Präferenzen der zuweisenden Ärzte, Patienten oder ihrer Familien. Hinterfragt wird dieses unsystematische Vorgehen nicht zuletzt deshalb, weil die Therapieerfolge in pädiatrischen Studien signifikant besser sind als in Erwachsenen-Studien. Es wurde diskutiert, ob es sich bei den Patienten in pädiatrischen Studien um solche mit einer günstigeren Prognose handelt, ob die Therapieprotokolle in der Pädiatrie effektiver sind oder ob schlicht die ärztliche Versorgung besser ist.

Die wesentliche Ursache liegt in der Tatsache, dass die Therapieergebnisse bei der ALL insgesamt stark altersabhän- gig sind. Innerhalb pädiatrischer Studien zeigt sich bereits eine Abnahme der Überlebensraten mit zunehmendem Alter. So nahm in der pädiatrischen BFM-95-Studie (BFM = Berlin-Frankfurt-Münster) die Gesamtüberlebensrate mit zunehmendem Lebensalter der Kinder ab und war bei den Jugendlichen über 15 Jahren am niedrigsten [3]. An diese Entwicklung schließen sich nahtlos die Erwachsenen-ALLStudien an. Hier sind die Gesamtüberlebensraten für die AYA am günstigsten und nehmen dann kontinuierlich ab. Die Gründe dafür sind vielfältig. Zum einen nehmen mit zunehmendem Alter ungünstige Prognosefaktoren zu. Zum anderen hängt der Therapieerfolg bei der ALL entscheidend von der zeitnahen Durchführung einer intensiven Chemotherapie ab. Die Therapiedurchführbarkeit und -verträglichkeit nehmen mit dem Alter kontinuierlich ab und daraus resultiert das schlechtere Therapieansprechen bei erwachsenen Patienten insgesamt und bei älteren Patienten im Besonderen.

In den vergangenen Jahren wurde eine Reihe von Studien an jungen Erwachsenen, die nach pädiatrischen Protokollen oder nach Protokollen für Erwachsene behandelt wurden, publiziert. Es zeigte sich, dass die Eingangskriterien für den Studieneinschluss vergleichbar waren und sich auch die Raten an Komplettremissionen nicht wesentlich voneinander unterschieden, jedoch war in den Pädiatrie-Studien das ereignisfreie Überleben (EFS) bzw. das krankheitsfreie Überleben (DFS) zum Teil deutlich länger [4]. Es fällt auf, dass die zum Vergleich herangezogenen Erwachsenen-Studien unterdurchschnittliche Ergebnisse erbracht hatten und zum Teil auf Therapieprinzipien beruhten, die nicht zeitgemäß sind. In neueren Vergleichen von Studien auf Basis innovativer Protokolle lassen sich keine deutlichen Unterschiede zwischen Pädiatrie- und Erwachsenen-Studien zeigen.

Es lohnt sich deshalb der Blick auf moderne ALL-Protokolle, wie sie etwa in der deutschen Studiengruppe für die ALL des Erwachsenen (GMALL) verwendet werden. Die aktuelle Studie GMALL 07/2003 wurde für Erwachsene und Adoleszente konzipiert und wird in 146 internistisch-onkologischen deutschen Zentren durchgeführt. Die Studien der

\section{KARGER}

Fax +497614520714

Information@Karger.de

www.karger.com (c) 2011 S. Karger GmbH, Freiburg

0378-584X/11/3417-0006\$38.00/0 
Tab. 1. Häufigkeit der ALL bei Adoleszenten (modifiziert nach [1])

\begin{tabular}{lr}
\hline & $\%$ \\
\hline Nicht gonadale Keimzelltumoren & 2 \\
Andere & 2 \\
Hodgkin-Lymphone & 12 \\
Non-Hodgkin-Lymphone & 7 \\
Melanome & 11 \\
Hodentumoren & 11 \\
Gynäkologische Tumoren & 10 \\
Schilddrüsen-CA & 10 \\
Weichteilsarkome & 8 \\
ZNS-Neoplasien & 6 \\
Leukämien & 6 \\
Andere Karzinome & 6 \\
Brustkrebs & 5 \\
Osteogone Tumoren & 3 \\
\hline
\end{tabular}

GMALL-Studiengruppe basieren ursprünglich, wie viele Erwachsenen-Studien, auf einem pädiatrischen Protokoll. Aufgrund eines Beschlusses des Gemeinsamen Bundesausschusses (GBA) werden seit einigen Jahren nur noch Patienten ab 18 Jahren aufgenommen.

Eine Zwischenanalyse dieser Studie bei AYA im Alter von 15-25 Jahren zeigt, dass bereits innerhalb der Gruppe der jungen Patienten der Anteil von Patienten mit ungünstigen Prognosefaktoren zunimmt. So ist etwa der Anteil von Patienten mit Höchstrisiko (Philadelphia-positive ALL) bei den über 20-Jährigen mehr als doppelt so hoch wie bei den 15- bis 17-jährigen Patienten. Die Gesamtergebnisse sind mit einer Remissionsrate von $91 \%$ und einer Gesamtüberlebensrate von $69 \%$ vielversprechend [5].

In den letzten Jahren wurden einige Studien veröffentlicht, in denen bei erwachsenen ALL-Patienten mehr oder weniger unverändert pädiatrische Therapieprotokolle eingesetzt wurden. Es handelte sich um kleine Studien mit bisher kurzer Nachbeobachtungszeit. Unter Beachtung dieser Einschränkungen waren die Ergebnisse zum Teil vielversprechend [6]. Es wurde jedoch eine erhöhte Inzidenz von Nebenwirkungen, z.B. Thrombosen und Neuropathien, berichtet. Die Mehrzahl der Studien wurde in einzelnen hoch spezialisierten Zentren durchgeführt, in denen auch eine sehr intensive Supportivtherapie angeboten werden kann.

Das wichtigste Ziel für die zukünftige Verbesserung der Therapieergebnisse bei AYA besteht darin, erfolgreiche Therapiekonzepte aus pädiatrischen Studien und ErwachsenenStudien zu kombinieren. Bei den Therapieelementen aus pädiatrischen Studien handelt es sich vorwiegend um chemotherapiebasierte Ansätze, dagegen wird die Stammzelltransplantation als intensivste Therapieform selten durchgeführt. Anders ist es bei den Erwachsenen-Studien, die die Rationale für die Therapie mit zielgerichteten Substanzen und modernen Transplantationskonzepten geliefert haben.

Pädiatrische Studien unterstreichen insbesondere die Bedeutung einer ohne Verzögerungen durchgeführten intensiven Chemotherapie. So wurde gezeigt, dass bei rezidivierten
Patienten jeder Tag Therapieverzögerung die Prognose verschlechtert [7]. Obwohl aus medizinischen Gründen bei erwachsenen Patienten Therapieverzögerungen manchmal nicht vermeidbar sind, muss auf eine konsequente Therapiedurchführung geachtet werden. Nicht zuletzt ist die pädiatrische Onkologie in deutlich weniger Zentren als die Erwachsenen-Onkologie organisiert. Dies führt dazu, dass in einzelnen Zentren mehr Erfahrungen mit der Erkrankung gesammelt werden können. Ein GBA-Beschluss zur Kinderonkologie macht verbindliche Vorgaben zur Ausstattung in solchen Zentren. Er fordert auch die Behandlung in Therapiestudien. Über $90 \%$ der pädiatrischen ALL-Patienten werden in klinische Studien eingebracht. Demgegenüber werden erwachsene ALL-Patienten derzeit in 146 Zentren behandelt. Wegen der Seltenheit der Erkrankung betreut eine ganze Reihe dieser Kliniken weniger als einen ALL-Patienten pro Jahr.

In den Studien der deutschen ALL-Studiengruppe wurden zahlreiche innovative Therapieansätze entwickelt. Dazu gehören auch eine intensive Chemotherapie und die Stammzelltransplantation bei Patienten mit Hochrisiko-Merkmalen. Weitere Therapiefortschritte wurden mit pegylierter Asparaginase in intensivierter Dosis als Erstlinientherapie erzielt, womit insbesondere die Remissionsdauer verlängert werden konnte [8].

Bei erwachsenen Patienten stößt die Chemotherapieintensivierung an Grenzen und der Einsatz zielgerichteter Substanzen kann die Effektivität verbessern, ohne die üblichen Nebenwirkungen zu verstärken. Auch für junge Erwachsene, die bereits mehr unter Nebenwirkungen der Chemotherapie leiden als Kinder, sind solche Therapieansätze relevant. So wurde unter anderem Rituximab bei CD20-positiver ALL erfolgreich geprüft [9]. Auch bei Patienten mit B-Zell-ALL (B-ALL) und Burkitt-Lymphomen hat sich die Überlebensrate mit Einführung von Rituximab als Therapiebaustein verbessert, wobei gleichzeitig die Methotrexat-Dosis und damit die Toxizität reduziert werden konnte [10]. Ein weiterer wichtiger Ansatz zur Therapieoptimierung, der in der GMALLStudiengruppe entwickelt wurde, ist die individualisierte Therapie basierend auf der minimalen Resterkrankung (MRD). Die Messung der MRD erlaubt es, das Therapieansprechen durch den Nachweis von Leukämiezellen auf sehr niedrigem Niveau (1 Leukämiezelle in 10000 normalen Zellen) sehr genau und individuell zu messen. ALL-Patienten, bei denen nach Induktion bzw. Konsolidierung noch eine MRD nachweisbar ist, weisen eine Chemotherapieresistenz auf und haben wegen der hohen Rezidivrate eine ungünstige Prognose [11]. Diese Patienten sollten transplantiert werden. Weiterhin besteht die Möglichkeit, die MRD durch experimentelle Therapien zu behandeln. Hoffnungsvoll stimmen Ergebnisse mit Blinatumomab, einem CD19-Antikörper. Er führte in einer Phase-II-Studie mit 20 ALL-Patienten mit anhaltendem Nachweis von MRD in $80 \%$ der Fälle zu einem Verschwinden der MRD. Einige Patienten hatten auch ohne Stammzelltransplantation lang anhaltende Remissionen [12]. 


\section{Fazit}

Die Ergebnisse der bisherigen ALL-Studien haben einige erfolgreiche Konzepte bestätigt, die auch für die Nachfolgestudie GMALL 08/2011 von Bedeutung sind. Die Studie wird durch eine weitere Intensivierung der Chemotherapie mit verbesserter Zeit- und Dosisintensität besonders die Gruppe der AYA in den Blick nehmen. Gleichzeitig werden besonders erfolgreiche individualisierte und zielgerichtete Therapieansätze weiterentwickelt. Neben der verbesserten Erstlinientherapie ist auch eine optimierte Rezidivtherapie von großer Bedeutung. Dies wird im Mittelpunkt eines neuen Projekts der GMALL-Studiengruppe stehen. Im Hinblick auf die Rahmenbedingungen der Therapie muss eine verbesserte Unterstützung der Patienten erfolgen, um die Therapie-Compliance zu verbessern. Hierfür ist eine verbesserte personelle Ausstattung erforderlich. Es muss diskutiert werden, ob es sinnvoll ist, eine seltene Erkrankung wie die ALL in einer großen Zahl von Kliniken zu behandeln. Um ähnlich wie in der Pädiatrie den Einschluss in klinische Studien zu verbessern, müssten die Rahmenbedingungen für Studien in Deutschland verbessert sowie Kliniken und Studiengruppen unterstützt werden.

Für die langfristige strategische Ausrichtung ist die Etablierung von Adoleszenten-Zentren, in denen Pädiater und Internisten interdisziplinär zusammenarbeiten, ein sinnvolles Ziel. Der Fokus sollte auf der psychosozialen Betreuung der Patienten liegen und im Mittelpunkt sollten dabei Tumorerkrankungen stehen, die bei AYA häufig auftreten. Dazu gehört nicht die ALL. Spezielle Therapieprotokolle für AYA werden wegen der geringen Patientenzahlen in den wenigsten Tumorentitäten sinnvoll sein. Für die ALL bei Adoleszenten und jungen Erwachsenen könnte die Verbindung von Ansätzen aus der Pädiatrie und aus Erwachsenen-Studien, wie sie in der nächsten GMALL-Studie geplant sind, eine weitere Verbesserung der Heilungsraten bringen.

\section{Hochmaligne Lymphome bei jungen Patienten \\ Verantwortliche Autoren: Gerald Wulf, Göttingen, Lorenz, Trümper, Göttingen}

Lymphome haben in der Gruppe der AYA (15-19 Jahre) einen Anteil von 26\% an der Gesamtzahl der Krebserkrankungen [13], wobei sich das Spektrum der histologischen Subtypen mit dem Lebensalter ändert. Bei Kindern (0-14 Jahre) sind Burkitt-Lymphome mit einem Anteil von 38\% der dominierende Subtyp der Non-Hodgkin-Lymphome (NHL), wogegen im Jugendalter (15-19 Jahre) die diffus großzelligen B-Zell-Lymphome (DLBCL) stetig an Bedeutung gewinnen [14]. Es handelt sich somit um eine sehr heterogene Erkrankung, deren Erscheinungsbild sich bei pädiatrischen und adoleszenten Patienten deutlich unterscheidet (Abb. 1). Dies wurde kürzlich auch mit Daten aus Deutschland belegt [15].

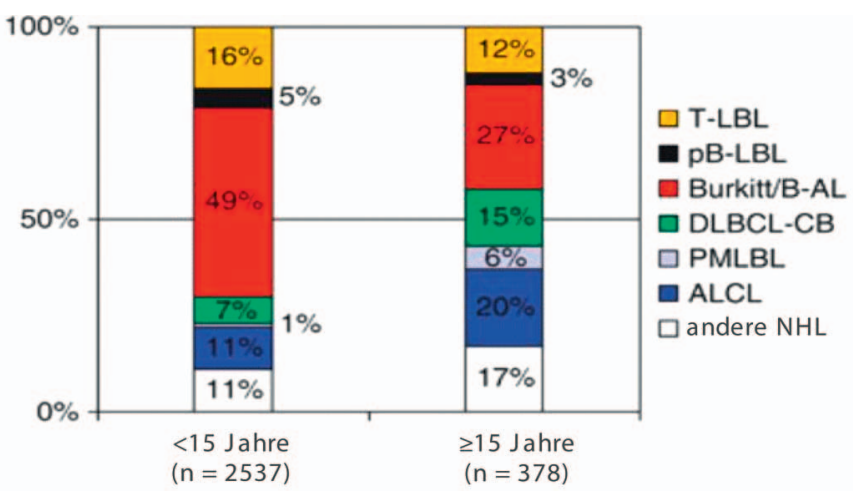

Abb. 1. Altersabhängige Verteilung der NHL-Subtypen (modifiziert nach [15]).

Die Therapie von Patienten mit aggressiven NHL orientiert sich am histologischen Subtypus des Lymphoms, wobei Kinder und Jugendliche fast ausschließlich im Rahmen von kontrollierten multizentrischen Therapiestudien behandelt werden. Ein Beispiel sind die nach histologischen Subtypen stratifizierten Therapieprotokolle NHL-BFM der Gesellschaft für Pädiatrische Onkologie und Hämatologie. Zu nennen sind hier die Studien B-NHL BFM 04 (multizentrische Beobachtungsstudie zur Behandlung von Kindern und Jugendlichen mit reifem B-Zell-NHL oder B-ALL) sowie die Studie B-NHL BFM Rituximab (multizentrische Therapiestudie mit einem Rituximab-Window vor anschließender Chemotherapie zur Behandlung von Kindern und Jugendlichen mit reifem B-Zell-NHL oder B-ALL).

Burkitt-Lymphome und lymphoblastische Lymphome werden wegen ihrer biologischen Verwandtschaft zur ALL erfolgreich nach ALL-vergleichbaren GMALL-Therapieprotokollen behandelt. Periphere T-Zell-Lymphome spielen im Kindesalter eine untergeordnete Rolle, weshalb die Etablierung von speziell pädiatrischen bzw. AYA-Therapiekonzepten nicht sinnvoll erscheint. Anders ist es bei den DLBCL und den primär mediastinalen B-Zell-Lymphomen (PMBCL), bei denen ein differenziertes Vorgehen in Abhängigkeit vom Lebensalter nötig und möglich ist.

In der Pädiatrie wird aufgrund der insgesamt geringen Patientenzahlen keine NHL-Therapiestratifizierung nach histologischen Subtypen vorgenommen, sondern durchgehend mit den NHL-BFM-Therapieprotokollen gearbeitet. Sowohl bei Kindern (unter 15 Jahre) als auch bei Adoleszenten (1519 Jahre) werden damit über den gesamten Bereich der hochmalignen NHL gute Ergebnisse erzielt; das 10-JahresGesamtüberleben beträgt $86 \%$ bei einem EFS von $79 \%$ [15]. Bei getrennter Auswertung der Patienten mit DLBCL und PMBCL fällt allerdings auf, dass das mediane EFS von Jugendlichen ab 15 Jahren gegenüber den jüngeren Kindern verkürzt ist. Dabei muss beachtet werden, dass in der gepoolten Analyse auch Daten von Patienten enthalten sind, die noch kein Rituximab erhalten hatten. Ein überraschendes Ergebnis der Datenauswertung von insgesamt 378 adoleszen- 
ten Patienten mit aggressivem NHL war außerdem, dass die männlichen Patienten $(\mathrm{n}=259)$ ein längeres 10-Jahres-Gesamtüberleben $(83 \%)$ erreichten als die weiblichen Patienten (70\%). Die Gründe dafür sind nicht bekannt, womöglich sind hormonelle Einflüsse und eine geschlechtsspezifische Pharmakokinetik für das unterschiedliche Outcome verantwortlich. So gibt es Hinweise, dass der Antikörper Rituximab bei männlichen und weiblichen Patienten unterschiedlich schnell metabolisiert wird und deshalb Dosisanpassungen in Abhängigkeit vom Geschlecht nötig sind.

AYA-Patienten mit hochmalignen NHL im Alter von 19-34 Jahren werden in der Regel nach den Therapieprotokollen der Deutschen Studiengruppe Hochmaligne NonHodgkin-Lymphome (DSHNHL) behandelt. Aus den DSHNHL-Studien der Jahre 1992-2008 überblicken wir mehr als 500 Patienten, die bei Therapiebeginn zur AYA-Altersgruppe gehörten. Ein größerer Teil von ihnen war Teilnehmer der MinT-Studie (Intergruppen-Studie für die First-Line-Behandlung von Patienten mit diffusem großzelligem B-Zell-NHL mit einem Cyclophosphamid/Hydroxydaunorubicin/Vincristin/Prednisolon (CHOP)-ähnlichen Chemotherapie-Behandlungsschema mit oder ohne anti-CD20-Antikörper Rituximab). Weitere Patienten hatten im Rahmen der NHL-B1-Studie das Therapieregime CHOP 21 erhalten. Die übrigen AYA-Patienten wurden nach verschiedenen Protokollen mit Cyclophosphamid/Hydroxydaunorubicin/Vincristin/Etoposid/ Prednisolon (CHOEP)-21 oder höher dosierten CHOEPRegimen behandelt. Die Unterteilung der AYA-Patienten bzw. jungen Erwachsenen in 4 Altersstufen zwischen 18 und 35 Jahren ergab in vorläufigen Analysen bisher keinen altersabhängigen Einfluss auf das Gesamtüberleben. Dies galt in ähnlicher Weise für das EFS. Auch bei gesonderter Betrachtung der Patienten mit DLBCL als größte Einzelgruppe in der Studienpopulation der AYA bestätigt sich, dass bei den bis zu 35-Jährigen das Alter prognostisch keine wesentliche Rolle spielt. Moderne Therapiekonzepte unter Hinzunahme von Rituximab haben allen Patienten einen Benefit gebracht, wobei innerhalb der Gruppe der AYA keine Unterschiede in der Effektivität beobachtet wurden. Wie bereits aus den älteren Studien vor Einführung von Rituximab bekannt, korreliert ein höheres Lebensalter (ab 50 Jahre) grundsätzlich mit einem schlechteren Therapieerfolg, weshalb die Gruppe der AYA gegenüber den älteren Patienten einen deutlichen Überlebensvorteil hatte. Bei $42 \%$ von 524 in der oben genannten Datenanalyse berücksichtigten AYA-Patienten war das DLBCL primär mediastinal lokalisiert. Das Risiko einer frühen Tumorprogression hat sich bei diesen Patienten mit Einführung von Rituximab als Therapiebaustein deutlich vermindert [16]. Dies hat zu vergleichbaren Überlebensraten bei DLBCL unabhängig von der primären Tumorlokalisation geführt.

DLBCL weisen auch hinsichtlich der molekularen Pathogenese erhebliche Unterschiede auf. Mithilfe von Genexpressionsanalysen ist eine Einteilung in die Subtypen GCB (ger- minal center B cell-like) und ABC (activated B cell-like) möglich. Im Kindesalter sind die prognostisch günstigeren GCB-Lymphome der dominierende Tumortyp, allerdings wurde bei Verwendung moderner Therapieprotokolle kein Unterschied im Gesamtüberleben im Vergleich zu Kindern mit Non-GCB-Lymphomen gefunden [17]. Derzeit leitet sich deshalb aus der molekular-histologischen Befundung des Tumors kein diesbezüglich differenziertes therapeutisches Vorgehen für AYA-Patienten ab.

Auch die Burkitt-Lymphome weisen ein breites morphologisches, immunhistologisches und genetisches Spektrum auf, das durch die molekulare Burkitt-Lymphom-Signatur präzisiert werden konnte. Auf Basis von Genexpressionsanalysen kann ein hoher Anteil der kindlichen DLBCL den BurkittLymphomen zugeordnet werden [18]. Auch weist die Pathway-Aktivierung von Onkogenen bei Kindern und Adoleszenten ein vergleichsweise homogenes Muster auf [19], was die Empfindlichkeit der Tumorzellen gegenüber Target-Therapeutika verstärken dürfte. Folglich könnten diese tumorbiologischen Besonderheiten Einfluss auf die künftige Therapie von AYA-Patienten mit hochmalignen NHL haben.

\section{Fazit}

Hochmaligne Lymphome sind bei jungen Patienten relativ selten und zudem histologisch sehr unterschiedlich aufgebaut. Die Patienten erhalten in der Regel eine Chemoimmunotherapie auf Basis von Rituximab plus CHOP (R-CHOP), die ein gutes Therapieansprechen mit einem hohen Anteil an Langzeitüberlebenden ausweist. Da speziell für die Gruppe der AYA konzipierte Studien nicht zu erwarten sind, wird sich die Arbeit eines AYA-Netzwerkes auf die kritische Durchsicht von Erwachsenen-Studien mit Blick auf die Subgruppe der AYA-Patienten konzentrieren. Ein therapeutischer Benefit für AYA kann womöglich durch Maßnahmen wie Steigerung der Rituximab-Dosis, Bestrahlung des TumorBulks, vermehrte Nutzung der Positronenemissionstomographie (PET)-Diagnostik und Etablierung von Therapieprotokollen mit neuen Substanzen erreicht werden.

\section{Hodgkin-Lymphome bei jungen Patienten \\ Verantwortlicher Autor: Peter Borchmann, Köln}

Das Hodgkin-Lymphom gehört heute aufgrund der stadienangepassten Therapie und den beständigen Verbesserungen von Chemo- und Radiotherapie zu den am besten zu behandelnden bösartigen Erkrankungen. Aufgrund der relativ niedrigen Inzidenz von 2-3 Hodgkin-Neuerkrankungen auf 100000 Personen sollte es möglich sein, allen Patienten eine optimale, individualisierte Therapie anzubieten. Eine gesonderte Betrachtung der Gruppe heranwachsender HodgkinPatienten (AYA) ist nur dann sinnvoll, wenn es zwischen ihnen und Erwachsenen Unterschiede in der Physiologie oder der Tumorbiologie gibt. Tatsächlich sind aber die Organfunk- 
tionen und die grundlegenden metabolischen, immunologischen und hämatologischen Prozesse bei Kindern ab einem Lebensalter von etwa 10 Jahren vergleichbar denen erwachsener Menschen [20]. Auch gibt es keine Hinweise, dass sich die Tumorbiologie der AYA von der erwachsener Patienten wesentlich unterscheidet, zumal das Spektrum der Tumorerkrankungen bei beiden weitgehend identisch ist. Deutlich von den AYA und den jungen Erwachsenen lassen sich allerdings die Kinder (0-14 Jahre) abgrenzen [21], bei denen Tumoren des blutbildenden Systems und des Zentralnervensystems (ZNS) dominieren.

Anhand der Patientendaten, die der Deutschen Hodgkin Studiengruppe (GHSG) zur Verfügung stehen, sind wir der Frage nachgegangen, ob Adoleszente andere Krankheitsoder Patientencharakteristika aufweisen als junge Erwachsene bis zu einem Alter von 45 Jahren - die Gruppe der noch älteren Erwachsenen haben wir bewusst ausgeklammert, da das Therapieansprechen und damit der Therapieerfolg im höheren Lebensalter grundsätzlich schlechter sind. Wir haben aus diesen Überlegungen heraus fast 4000 Patienten herangezogen, die zwischen 1988 und 1998 in Studien behandelt wurden, wobei in jenen Jahren bereits Patienten ab 15 Jahren (heute: 18 Jahre) in Studien aufgenommen wurden. Für den Vergleich standen die Daten von 557 Adoleszenten (15-20 Jahre) und 3228 Erwachsenen (21-45 Jahre) zur Verfügung. Mögliche Altersgruppeneffekte wurden mittels proportionaler Cox-Risikoanalyse ermittelt; dazu wurden als Vergleichsparameter die Freedom from Treatment Failure (FFTF)-Rate nach einer medianen Beobachtungsdauer von 81 Monaten gewählt sowie die Gesamtüberlebensrate nach einer medianen Beobachtungsdauer von 85 Monaten.

Die Patientencharakteristika der Adoleszenten und jungen Erwachsenen waren vergleichbar, insbesondere waren die verschiedenen Tumorstadien in beiden Gruppen ähnlich verteilt [22]. Auffällig war lediglich, dass es unter den jungen Erwachsenen etwas mehr männliche Patienten (56\%) gab, wogegen das Geschlechterverhältnis bei den Adoleszenten ausgeglichen war. Unsere Datenanalyse liefert in der Gesamtschau keinen Hinweis darauf, dass es zwischen Adoleszenten und jungen Erwachsenen wesentliche Unterschiede in der Biologie der Hodgkin-Lymphome gibt. Bezüglich der Risikofaktoren fanden sich bei den Adoleszenten ein etwas höherer Anteil von Patienten mit einer großen mediastinalen Tumormasse als bei den Erwachsenen (30\% vs. 20\%) und mehr Patienten mit einem Befall von $\geq 3$ Lymphknoten (68\% vs. 59\%).

Des Weiteren stellt sich die Frage, ob Adoleszente schlechter auf eine Therapie ansprechen als junge Erwachsene. Dies lässt sich beim Vergleich der FFTF-Raten nicht feststellen, lediglich nach 10 Jahren sind die Heranwachsenden mit einer FFTF-Rate von $80 \%$ gegenüber den jungen Erwachsenen (FFTF 76\%) tendenziell im Vorteil [22]. Bei ähnlichem Therapieansprechen (Tab. 2) zeigt sich beim 10-Jahres-Gesamtüberleben ebenfalls ein leichter Vorteil von $92 \%$ vs. $87 \%$ zugunsten der jüngeren Vergleichsgruppe.
Tab. 2. Therapieansprechen von Adoleszenten im Vergleich zu jüngeren Erwachsenen (modifiziert nach [22])

\begin{tabular}{lcc}
\hline Therapieansprechen & Alle Stadien & \\
\cline { 2 - 3 } & Jugendliche, \% & junge Erwachsene, \% \\
\hline CR/CRu & 92,1 & 91,2 \\
PR & 1,8 & 1,9 \\
NC & 0,2 & 0,2 \\
Progression & 4,5 & 5,4 \\
Keine Angaben & 1,4 & 1,3 \\
\hline Gesamt & 100,0 & 100,0 \\
\hline
\end{tabular}

Auch andere Arbeitsgruppen [23] haben keine Unterschiede im progressionsfreien Überleben (PFS) und im Gesamtüberleben zwischen Adoleszenten (16-21 Jahre) und jungen Erwachsenen (22-45 Jahre) gefunden. Es bleibt deshalb festzuhalten, dass die Therapie-Response bei jugendlichen Patienten nicht schlechter ausfällt als bei älteren. Die Unterschiede im Gesamtüberleben dürften darauf zurückzuführen sein, dass onkologische Therapien langfristig besser toleriert werden, je jünger die Patienten sind.

Auch von den Nebenwirkungen einer spezifischen Hodgkin-Therapie ist die Gruppe der 15- bis 20-Jährigen nicht stärker betroffen als die der 21- bis 45-Jährigen, so ein weiteres Ergebnis der retrospektiven Auswertung auf Basis der GHSG-Daten. Das Risiko für Zweitmalignome ist in beiden Altersgruppen in etwa gleich und deutlich geringer im Vergleich zu den älteren Erwachsenen (46-75 Jahre). Als Zweitmalignome treten in den beiden jüngeren Altersgruppen vorrangig akute Leukämien, NHL und solide Tumoren auf, dabei weist das Erkrankungsspektrum keine altersabhängigen Differenzierungen auf.

Was die Therapiestrategien betrifft, so ist ein direkter Vergleich zwischen Kinder- und Erwachsenen-Protokollen aufgrund unterschiedlicher Konzepte kaum möglich. Bei einem indirekten Vergleich der Überlebensraten schneiden beide Strategien gut ab. Dabei ist zu berücksichtigen, dass sich die Staging-Systeme der GHSG von denen der Gesellschaft für Pädiatrische Onkologie und Hämatologie (GPHO) etwas unterscheiden. So werden in diesen empirischen Systemen erwachsene Patienten bei identischer Tumorausbreitung einem höheren Tumorstadium zugeordnet als Kinder - würde bei den Erwachsenen das Staging nach dem GPHO-System erfolgen, würden sie zum Teil mit weniger intensiver, zum Teil mit intensiverer Therapie behandelt. Dies verdeutlicht, dass ein indirekter Vergleich der Studien relevante systemische Fehler enthalten muss und daher nicht weiterführt.

Unsere Analyse hat Limitierungen: So verfügen wir zum jetzigen Zeitpunkt nur über eine limitierte Anzahl von AYA, die nach dem BEACOPP-Schema (Bleomycin, Etoposid, Adriamycin, Cyclophosphamid, Vincristin (= Oncovin), Prednisolon, Procarbazin) als Standard-Therapieprotokoll für die fortgeschrittenen Stadien beim Hodgkin-Lymphom behandelt worden sind. Auch fehlen für einen Vergleich der Therapie- 
effektivität zwischen den jüngeren und etwas älteren Patienten langfristige Daten zur Sicherheit, die mit der vorliegenden Analyse nicht erfasst werden.

\section{Fazit}

Die Biologie der Hodgkin-Lymphome unterscheidet sich bei heranwachsenden Patienten (ab 15/16 Jahre) nicht von denen bei jungen Erwachsenen, auch das Ansprechen auf die gewählte Therapie ist nahezu gleich. Dies bedeutet für die Betreuung von Hodgkin-Patienten sowie für die Konzeption künftiger Studien: Das erste Ziel bei der Behandlung des Hodgkin-Lymphoms bleibt die Heilung, sie sollte - zweites Ziel - mit so wenig Nebenwirkungen wie möglich erreicht werden. Dieser Anspruch beschränkt sich nicht auf eine bestimmte Gruppe von Patienten, sondern gilt für alle. Innerhalb unserer Protokolle haben die Adoleszenten und jungen Erwachsenen ein besonders gutes Outcome. Dennoch gibt es altersspezifische Besonderheiten, die eine Herausforderung darstellen: Für sehr junge Patienten gilt dies vor allem für die therapiebegleitenden Maßnahmen, insbesondere die psychosoziale Betreuung.

\section{Disclosure Statement}

Es bestehen keine Interessenkonflikte.

\section{Literatur}

1 Bleyer WA, Barr RD: Cancer in Adolescents and Young Adults. Berlin, Springer, 2007.

2 www.seer.cancer.gov.

3 Möricke A et al.: Alter als prognostischer Faktor bei Kindern und Jugendlichen mit akuter lymphoblastischer Leukämie: Ergebnisse aus den Therapiestudien ALL-BFM 86, 90 und 95. Klin Padiatr 2005;217:310-320.

4 Stock $\mathrm{W}$ et al.: What determines the outcomes for adolescents and young adults with acute lymphoblastic leukemia treated on cooperative group protocols? A comparison of Children's Cancer Group and Cancer and Leukemia Group B studies. Blood 2008;112:1646-1654.

$\checkmark 5$ Gökbuget $\mathrm{N}$ et al.: Risk adapted treatment of adolescents with acute lymphoblastic leukemia (ALL) according to the German Multicenter Study Group (GMALL) studies 06/99 and 07/03 yields significantly different outcome for subgroups. Haematologica 2007;92:135.

6 Huguet F et al.: Pediatric-inspired therapy in adults with Philadelphia chromosome-negative acute lymphoblastic leukemia: the GRAALL-2003 study. J Clin Oncol 2009;27:911-918.

7 Herold R et al.: Acute lymphoblastic leukemiarelapse study of the Berlin-Frankfurt-Munster Group (ALL-REZ BFM) experience: early treatment intensity makes the difference. J Clin Oncol 2004;22:569-570.

8 Gökbuget $\mathrm{N}$ et al.: PEG-asparaginase intensification in adult acute lymphoblastic leukemia (ALL) Significant improvement of outcome with moderate increase of liver toxicity in the German Multicenter Study Group for Adult ALL (GMALL) study 07/2003. Blood (ASH Annual Meeting Abstracts) 2010;116:abstr 494 .
9 Hoelzer et al.: Immunochemotherapy with rituximab improves molecular CR rate and outcome in CD20+ B-lineage standard and high risk patients; results of $263 \mathrm{CD} 20+$ patients studied prospectively in GMALL study 07/2003. Blood (ASH Annual Meeting Abstracts) 2010;116:abstr 170.

10 Hoelzer D et al.: High survival rate in adult Burkitt's lymphoma/leukemia and diffuse large Bcell lymphoma with mediastinal involvement. Blood (ASH Annual Meeting Abstracts) 2007; 110:abstr 518 .

11 Gökbuget $\mathrm{N}$ et al.: New definition of treatment response in adult acute lymphoblastic leukemia (ALL): Use of molecular markers for minimal residual disease (MRD). Blood (ASH Annual Meeting Abstracts) 2009;112:abstr 90.

12 Topp $M$ et al.: CD19/CD3 bispecific antibody blinatumomab (MT-103) is highly effective in treatment of patients with minimal residual disease from chemotherapy-resistant B-precursor acute lymphoblastic leukemia. Blood (ASH Annual Meeting Abstracts) 2010;116:abstr 174.

13 Bleyer WA et al.: Cancer Epidemiology in Older Adolescents and Young Adults 15-29 Years of Age, Including SEER Incidence and Survival, 1975-2000. Bethesda, National Cancer Institute, 2006, NIH Pub. No. 06-5767.

14 Hochberg J et al.: Adolescent non-Hodgkin lymphoma and Hodgkin lymphoma: state of the science. Brit J Haematol 2009;144:24-40.

15 Burkhardt B et al.: Non-Hodgkin's lymphoma in adolescents: experiences in 378 adolescent NHL patients treated according to pediatric NHL-BFM protocols. Leukemia 2011;25:153-160.
16 Rieger M et al.: Primary mediastinal B-cell lymphoma treated with CHOP-like chemotherapy with or without rituximab: results of the Mabthera International Trial Group study. Ann Oncol 2011;22:664-670.

17 Oschlies I et al.: Diffuse large B-cell lymphoma in pediatric patients belongs predominantly to the germinal-center type B-cell lymphomas: a clinicopathologic analysis of cases included in the German BFM (Berlin-Frankfurt-Munster) Multicenter Trial. Blood 2006;107:4047-4052.

18 Klapper W et al.: Molecular profiling of pediatric mature B-cell lymphoma treated in populationbased prospective clinical trials. Blood 2008;112: 1374-1381.

19 Bentink S et al.: Pathway activation patterns in diffuse large B-cell lymphomas. Leukemia 2008;22: $1746-1754$.

20 Conners J: Workshop on HL in adolescents: a controversy? 8th International Symposium on Hodgkin Lymphoma, October 23-26, Cologne, 2010

21 Bleyer A: Young adult oncology: the patients and their survival challenges. CA Cancer J Clin 2007;57:242-255.

22 Eichenauer DA et al.: Hodgkin's lymphoma in adolescents treated with adult protocols: a report from the German Hodgkin Study Group. J Clin Oncol 2009;27:6079-6085.

23 Foltz LM et al.: Hodgkin's lymphoma in adolescents. J Clin Oncol 2006;24:2520-2526. 